\title{
Molecular dynamics of electrosprayed water nanodroplets containing sodium bis(2-ethylhexyl)sulfosuccinate
}

\author{
Giovanna Longhi, ${ }^{a, b}$ Alberto Ceselli, ${ }^{c}$ Sandro L. Fornili, ${ }^{c}$ Sergio Abbate, ${ }^{a, b}$ \\ Leopoldo Ceraulo $^{\mathrm{d}, \mathrm{e}}$ and Vincenzo Turco Liveri $^{\mathrm{d}_{*}}$
}

\begin{abstract}
The behavior of aqueous solutions of sodium bis(2-ethylhexyl)sulfosuccinate (AOTNa) subject to electrospray ionization (ESI) has been investigated by molecular dynamics (MD) simulations at three temperatures (350, 500 and $800 \mathrm{~K}$ ). We consider several types of water nanodroplets containing AOTNa molecules and composed of a fixed number of water molecules (1000), $N_{A O T}^{O}$ AOT $^{-}$anions $\left(N_{A O T}^{O}=0,5,10\right)$ and $N_{N a}^{o}$ sodium ions $\left(N_{N a}^{o}=0,5,10,15,20\right)$ : in a short time scale (less than 1 ns), the AOTNa molecules, initially forming direct micelles in the interior of the water nanodroplets, are observed in all cases to diffuse nearby the nanodroplet surface, so that the hydrophilic heads and sodium ions become surrounded by water molecules, whereas the alkyl chains lay at the droplet surface. Meanwhile, evaporation of water molecules and of solvated sodium ions occurs, leading to a decrease of the droplet size and charge. At $350 \mathrm{~K}$, no ejection of neutral or charged surfactant molecules is observed, whereas at $500 \mathrm{~K}$, some fragmentation occurs, and at $800 \mathrm{~K}$, this event becomes more frequent. The interplay of all these processes, which depend on the values of temperature, $N_{A O T}^{o}$ and $N_{N a}^{o}$ eventually leads to anhydrous charged surfactant aggregates with prevalence of monocharged ones, in agreement with experimental results of ESI mass spectrometry. The quantitative analysis of the MD trajectories allows to evidence molecular details potentially useful in designing future ESI experimental conditions. Copyright @ 2013 John Wiley \& Sons, Ltd.
\end{abstract}

Supporting information may be found in the online version of this article.

Keywords: AOTNa; electrospray ionization; aqueous nanodroplets; charged reverse micelle-like aggregates; molecular dynamics simulation

\section{Introduction}

Electrospray ionization (ESI) is a soft technique widely employed in mass spectrometry allowing to generate multiply charged high-molecular weight molecular and/or supramolecular species with minimal chemical decomposition of the molecules under study. In typical ESI-MS experiments, highly charged micrometersized droplets are ejected from the tip of the Taylor cone in the surrounding gas phase and are driven by the external electric field towards the counter-electrode. ${ }^{[1-4]}$

During this flight, some out-of-equilibrium processes take place. In particular, the droplet may undergo collisions with surrounding gas molecules and other droplets, and thus evaporation of neutral volatile components (generally, solvent molecules) and/or Rayleigh instability may occur, leading to the emission of charged nanodroplets and ejection of solvated ions. ${ }^{[5-7]}$

These interconnected phenomena are accompanied by changes in the droplet size and charge state as well as in the radial distribution of ionic species within the droplet. It is generally believed that while solvent evaporation and ion spatial rearrangement begin when the droplet is formed, ejection of solvated ions takes place when the charge density reaches a critical value. ${ }^{[8]}$ Solvent molecules and solvated ions are preferentially released from the tips of transient protrusions originated by local instabilities of the droplet surface. ${ }^{[9]}$ Thus, the species observed by mass spectrometry are produced through evaporation of volatile components and ejection of charged ions. These processes are strongly impacted by experimental conditions (i.e. nature and composition of the electrosprayed solution, temperature of the tip and surrounding gas phase and external electric field).

Even though each one of these processes is amenable to a qualitative description, an overall picture is hard to come about, especially at the quantitative level. Together with experimental findings for droplets in the micrometer-size regime, ${ }^{[10]}$ a great deal of detailed and sometimes unexpected information on such processes has been gained by molecular dynamics (MD) simulations especially for nano-sized droplets. $^{[9,11,12]}$

\footnotetext{
* Correspondence to: Vincenzo Turco Liveri, Dipartimento STEBICEF, Universitò di Palermo, Viale delle Scienze Parco d'Orleans II, 90128 Palermo, Italy. E-mail: vincenzo.turcoliveri@unipa.it

a Dipartimento di Scienze Biomediche e Biotecnologie, Università di Brescia, Viale Europa 11, 25123 Brescia, Italy

b CNISM, Consorzio Interuniversitario Scienze Fisiche della Materia, Via della Vasca Navale 84, 00146 Roma, Italy

c Polo Didattico e di Ricerca di Crema, Universita' di Milano, Via Bramante 65, 26013 Crema CR, Italy

d Dipartimento STEBICEF, Università di Palermo, Via Archirafi 32, 90123 Palermo, Italy

e Centro Grandi Apparecchiature, UniNetLAb, Via F. Marini 14, 90128 Palermo, Italy
} 
The most striking results of these studies for aqueous nanodroplets containing simple ionic species are:

(i) ions in excess are not located at the droplet surface as expected in terms of continuum electrostatic theory, but are found in a thick shell below the surface leading to complete ion solvation. This peculiar ion distribution involves some ordering of solvent molecules at the droplet surface. ${ }^{[11]}$

(ii) Shrinkage of nanodroplets, due to water evaporation, occurs through the formation of thorns originated by shape fluctuations. ${ }^{[9,13]}$

(iii) Ejection of each one of the solvated ion through a newly formed water thorn occurs when a critical value of the charge density is reached. It has been observed that the frequency of occurrence of local instabilities preceding thorn formation increases with the number of extra charges. ${ }^{[9,13]}$

(iv) Solvent evaporation and solvated ion ejection from aqueous nanodroplets are less pronounced than from methanol and water/methanol ones. Incidentally, in the case of solvent mixtures, the occurrence of partial solvent de-mixing is observed. ${ }^{[9,13]}$

Of course, it can be expected that some peculiar departures from the above description may occur when the electrosprayed aqueous solutions contain surfactant molecules. Indeed, above the critical micellar concentration (CMC), surfactant molecules in water associate leading to the formation of direct micelles, which are globular aggregates characterized by an internal core formed by the surfactant alkyl chains and a surrounding layer constituted by its hydrated head groups. Besides, at the water/air interface, a monolayer is formed by surfactant molecules oriented so that the polar head groups are immersed in the liquid phase while the alkyl chains protrude toward the gas phase.

The formation of oriented monolayers of surfactant molecules at the surface of water droplets is of utmost importance, since it determines a marked decrease of the droplet surface tension as well as changes of the evaporation rates and net charge spatial distribution. ${ }^{[14]}$

In particular, taking into account the Rayleigh equation, ${ }^{[15]}$ which enables one to determine the maximum charge $\left(Q_{\max }\right)$ sustainable by a droplet of radius $R$

$$
\mathrm{Q}_{\max }=8 \pi\left(\varepsilon_{0} \gamma \mathrm{R}^{3}\right)^{1 / 2}
$$

(where $\varepsilon_{0}$ is the permittivity of vacuum, and $\gamma$ is the surface tension of the solvent), we can evaluate the ratio $\mathrm{Q}_{\max , S} / \mathrm{Q}_{\max , \mathrm{w}}$ between the maximum charge in the presence of a surfactant layer $\left(Q_{\max , S}\right)$ and that in pure water $\left(\mathrm{Q}_{\max , \mathrm{w}}\right) \cdot \mathrm{Q}_{\max , \mathrm{S}} / \mathrm{Q}_{\max , \mathrm{W}}=0.7$ is obtained by considering $\gamma_{w}=72.8 \mathrm{mNm}^{-1}$ for pure water at room temperature and $\gamma_{\mathrm{S}}=40 \mathrm{mNm}^{-1}$ for a typical surfactant solution above the CMC, indicating that the maximum charge which can be carried by a surfactant-covered water droplet is $30 \%$ lower than that for bare water droplet. $^{[16]}$

Moreover, recent MD simulations have shown that a nanodroplet consisting of water molecules surrounding a direct micelle formed by cetyl trimethylammonium bromide molecules reverts to a water-containing reverse micelle in about $1-3 \mathrm{~ns}^{[17,18]}$ This time scale is much smaller than the residence time of aggregates within the mass spectrometer, which is of the order of $\mathrm{ms}$.

In view of the recent interest for charged surfactant aggregates in the gas phase $\mathrm{e}^{[19-24]}$ and in order to gain a more quantitative knowledge of the behavior of aqueous surfactant solutions under ESI conditions, we decided to carry out MD simulations of water nanodroplets containing the surfactant sodium bis(2-ethylhexyl) sulfosuccinate (AOTNa). AOTNa is a well-known di-chained surfactant (see Fig. 1) able to form direct micelles in water above its $C M C$, which is in the range $2.5-610^{-3} \mathrm{M}^{[25]}$ Water has been chosen as solvent because, due to the significant reduction of the surface tension induced by the surfactant, it works very well during ESI. ${ }^{[26]}$ Moreover, the data analysis is simpler than for the case of solvent mixtures, since one does not need to take into account phenomena as different evaporation rates for each solvent, different distributions of solvent molecules, preferential solvation of surfactant head groups and counterions.

Since a countercurrent sheath gas at atmospheric pressure is generally used allowing to adjust nanodroplet temperature over a wide range $(350-800 \mathrm{~K}),{ }^{[2,4,27,28]}$ we studied the time evolution of water nanodroplets containing AOTNa by carrying out simulations at three temperatures spanning the entire range $(350,500,800 \mathrm{~K})$. It is worth to note that the range of simulation temperatures has been chosen not only to deal with temperatures used in standard experiments, but also to explore the expected system behavior even in less usual conditions. The investigated systems consist of nanodroplets including a fixed number of water and variable numbers of $\mathrm{AOT}^{-}$and of $\mathrm{Na}^{+}$ions. Preliminarily, simulations were performed to test the behavior of the nanodroplet under an external electric field of $5 \mathrm{kV} / \mathrm{cm}$, typical of electrospray experiments. ${ }^{[1]}$ No significant change on the structural and dynamic properties of nanodroplets was observed from zero electric field condition; thus all simulations were performed by turning off the external electric field. Indeed, significant droplet deformations have been observed above a critical electric field of the order of $5000 \mathrm{kV} / \mathrm{cm}$, i.e. three order of magnitude larger than that typically employed for ESI. ${ }^{[5,7]}$ Moreover, replicas of the same simulation show some statistical variability but the key features are retained.

\section{Computational Methods}

The $\mathrm{AOT}^{-} R-R-R$ diastereoisomer, whose structure and atom labelling are reported in Fig. 1 for ease of description, was modelled according to the all-atom General Amber Force Field ${ }^{[29]}$ as previously described ${ }^{[23]}$ where satisfactory performance had been obtained. In particular, charges had been determined using the RESP protocol ${ }^{[30]}$ to comply with the AMBER force field, following quantum mechanical geometry optimization of molecular conformations at the RHF/6-31G* level. ${ }^{[23]}$
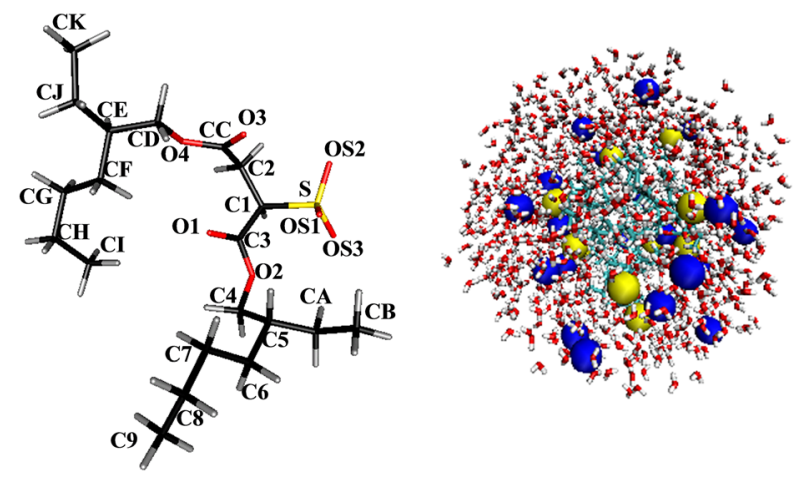

Figure 1. Left: structure (and atom labelling) of the $\mathrm{AOT}^{-}$anion in the conformational minimum obtained by previous ab-initio calculations. ${ }^{[23]}$ Right: snapshot of the $[10,20]$ system (aggregate of $10 \mathrm{AOT}^{-}, 20 \mathrm{Na}^{+}$ions and 1000 water molecules) after simulation under periodic boundary at $300 \mathrm{~K}$ : sodium ions (blue) and $\mathrm{SO}_{3}^{-}$polar heads (red oxygen atoms and yellow sulfur atoms) are displayed in a space-filling mode to evidence their location. This figure is available in colour online at wileyonlinelibrary.com/journal/jms 
The simulated systems were built using the LeAP module of Amber Tools 1.4. ${ }^{[31]}$ They consist of $N_{\text {AOT }}^{0}$ AOT ${ }^{-}$anions $\left(N_{A O T}^{0}=0,5,10\right)$, $N_{\mathrm{Na}}^{0} \mathrm{Na}^{+}$ions $\left(N_{\mathrm{Na}}^{0}=0,5,10,15,20\right)$ immersed into a truncated octahedral box of 1000 TIP3P waters. ${ }^{[32]}$ Each system was initially simulated under periodic boundary conditions (PBC) for $1 \mathrm{~ns}$ at $300 \mathrm{~K}$ and 1 bar using the SANDER module of the AMBER 10 package. ${ }^{[31,33]}$ The use of PBC implies that an extended surfactantcontaining aqueous phase is simulated. During this simulation stage, a 9-Å cut-off value and particle mesh Ewald were adopted for the non-bonded interactions. SHAKE ${ }^{[34]}$ kept bonds involving hydrogen atoms constrained. After this stage, each system is treated like a nanodroplet. Indeed, the final configurations obtained under PBC were assumed as the initial configurations of the vacuum simulations carried out using the NAMD 2.7 program $^{[35]}$ with the AMBER force field option. Constant temperature was enforced using Langevin dynamics with a damping coefficient of $1 \mathrm{ps}^{-1}$. No cutoff was applied for non-bonded interactions (more precisely, a 999- $\AA$ cutoff was used), and 2 fs time step was used. Vacuum simulations at constant temperature have been chosen as a computationally affordable way to realistically approximate the nanodroplet behavior under ESI conditions. ${ }^{[18]}$

The statistical analysis of the trajectories was mainly based on the PTRAJ module of the Amber Tools 1.4 package. Graphical analysis was performed using VMD. ${ }^{[36]}$ Molecular volumes have been evaluated by the Mol_Volume program developed by A. Balaeff. ${ }^{[37]}$

Some analysis was preceded by a search of aggregates performed by a home-made procedure exploiting graph theoretical tools. In particular, the graph considers one vertex for each ion (ion vertex), one vertex for each oxygen atom of the water molecules (water vertex) and one vertex for each AOT $^{-}$polar head (AOT vertex). Furthermore, one edge is included between each pair of vertices according to a matrix of parameters describing the interactions among water, sodium ions and AOT $^{-}$anions. Then, a search for connected components in this graph, representing aggregates in the MD simulation snapshot, is carried out using a breadthfirst-search (bfs) algorithm. ${ }^{[38]}$ The software parsing the snapshots, implementing the bfs algorithm and reporting statistics on the aggregates, has been coded in $\mathrm{C}++$, awk and shell script.

\section{Results and discussion}

The systems on which MD simulations were carried out consist of aqueous nanodroplets with 1000 water molecules, $N_{\text {AOT }}^{0}$ AOT $^{-}$ anions and $N_{\mathrm{Na}}^{0}$ sodium ions and will be indicated hereafter as $\left[N_{\mathrm{AOT}}^{0}, N_{\mathrm{Na}}^{0}\right]$. Their behavior has been investigated at three temperatures $(350,500,800 \mathrm{~K})$. As described in the previous section, the starting configuration was obtained by randomly placing the surfactant molecules within a box of water to perform preliminary liquid phase simulations under PBC at $300 \mathrm{~K}$. As expected, at the end of this preparation step, the surfactant molecules aggregated as direct micelle surrounded by sodium ions and water molecules according to their typical self-assembling pattern in highly polar media. This is exemplified by Fig. 1 where we report a typical configuration obtained by this procedure for the $[10,20]$ system. The configurations prepared in this way are then used to initiate the corresponding vacuum simulations.

Placing the nanodroplets in vacuo, some out-of-equilibrium processes take place, under the strong influence of temperature and nanodroplet composition. We first concentrate our discussion on the case $[10,20]$ which has revealed as the most instructive one. Typical snapshots of aqueous-AOTNa nanodroplets at selected successive times are shown in Fig. 2 where only ions and molecules still present in the nanodroplet are displayed. The system evolution at the three temperature values is markedly different.

Looking at Fig. 2A, B and C, some preliminary qualitative observations can be made. The first frame of each row, taken immediately after equilibration, shows that the nanodroplet contains a direct micelle surrounded by water: the aliphatic chains constitute the core of the aggregate which has a spherical shape, the surfactant heads $\mathrm{SO}_{3}^{-}$point outward, the $\mathrm{Na}^{+}$ions are dispersed throughout the external water layer. It can be noted that, while at $350 \mathrm{~K}$, the nanodroplets retain a large fraction of the water molecules present initially for comparatively a long time; at $500 \mathrm{~K}$ and $800 \mathrm{~K}$, they rapidly lose water inducing the formation of completely or practically anhydrous aggregates.

Moreover, the first $\mathrm{Na}^{+}$ion is ejected at ca. $\mathrm{t}=0.3 \mathrm{~ns}$, for $\mathrm{T}=350 \mathrm{~K}$, and at $\mathrm{t}=0.03 \mathrm{~ns}$ for $\mathrm{T}=500 \mathrm{~K}$, while the aggregates still show a direct-micelle structure and many water molecules are present. During subsequent times, the droplets keep losing water molecules and $\mathrm{Na}^{+}$ions, thus progressively lowering their net charge, while AOTNa molecules change their aggregation structure. On the contrary, at $800 \mathrm{~K}$, immediate ejection of $\mathrm{Na}^{+}$ ions and water molecules occurs at the very beginning of the simulation (at $0.01 \mathrm{~ns}$ the first $\mathrm{Na}^{+}$ion has already left the droplet). It can also be noted that $\mathrm{Na}^{+}$ions exit the droplet being surrounded by a crown of water molecules.

At $\mathrm{T}=350 \mathrm{~K}$, the inversion of the micelle-like structure occurs while water is still present in the nanodroplet in large amounts: the surfactant anions and their counterions keep close to each other, and this takes place through clustering of the hydrophobic tails in a region close to the droplet surface, while many $\mathrm{Na}^{+}$ions are dissolved in the bulk water of the droplet itself. At about $\mathrm{t}=0.5 \mathrm{~ns}$, aliphatic tails definitely point outward the droplet. This situation persists for the rest of the simulation, notwithstanding intermittent perturbations caused by ejection of water molecules and sodium ions.

At $\mathrm{T}=500 \mathrm{~K}$, a different behavior is observed: the system loses water molecules quite rapidly, and this strong ejection hampers self-organization of surfactant molecules to some extent: not only aggregation is made difficult by high temperature, but the whole system itself appears to start to break apart (see snapshot at $\mathrm{t}=0.34 \mathrm{~ns}$ ), but later surfactant molecules reaggregate at a time comparable to that of the $\mathrm{T}=350 \mathrm{~K}$ case. Indeed, at about $\mathrm{t}=0.5 \mathrm{~ns}$, the aliphatic tails point outward, some water is still present and one may speak of reverse micelle-like aggregate. Residual water is eliminated subsequently, without breaking the surfactant cluster; at $\mathrm{t}=0.67 \mathrm{~ns}$, one can observe the last $\mathrm{Na}^{+}$ion ejection. Thus, higher temperature causes faster ejection of water molecules and $\mathrm{Na}^{+}$ions, but this does not speed up significantly the micelle rearrangement.

At $\mathrm{T}=800 \mathrm{~K}$, the system changes are very rapid, and the droplet blows up: in the absence of enough water molecules, surfactant molecule aggregates cannot organize the hydrophobic tails (see for example $\mathrm{t}=0.05 \mathrm{~ns}$ ) and thus cannot give rise to a unique core of charged species, but instead give rise to multiple little and quite anhydrous surfactant aggregates (see $t=0.1 \mathrm{~ns}$ and subsequent times).

As stated before, even at $350 \mathrm{~K}, 1 \mathrm{~ns}$ time is enough to allow surfactant molecules to reshuffle within the aggregate, so that the hydrophilic heads and sodium ions get surrounded by water molecules whereas the alkyl chains lay at the droplet surface. Moreover, water evaporation occurs through alignment of water molecules in segments protruding outwards, whereas sodium 


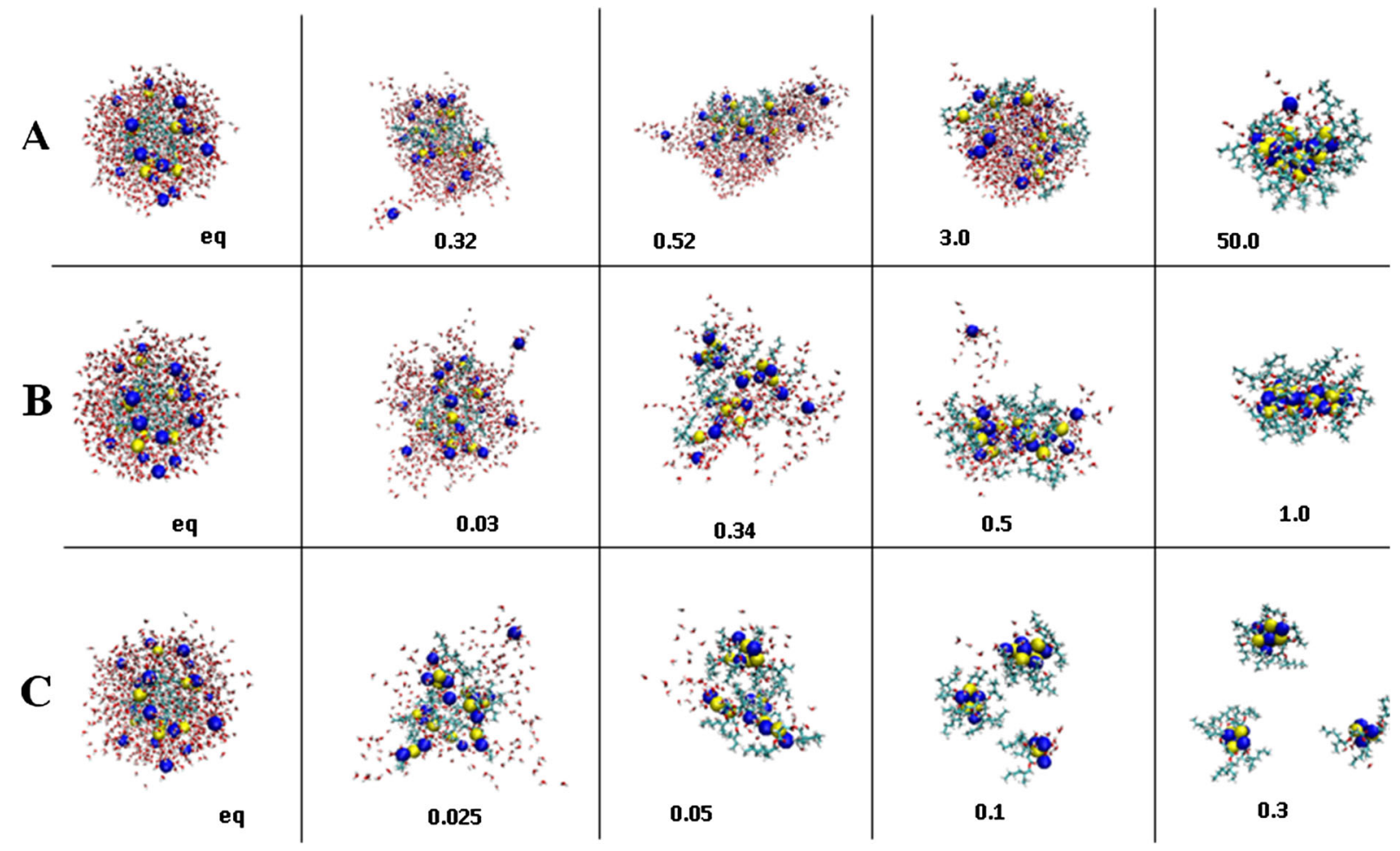

Figure 2. Snapshots of the $[10,20]$ system at $350 \mathrm{~K}(\mathrm{~A}), 500 \mathrm{~K}(\mathrm{~B}), 800 \mathrm{~K}(\mathrm{C})$ at selected simulation times (ns). Sodium ions (blue) and sulfur atoms of $\mathrm{SO}_{3}^{-}$polar heads (yellow) are displayed in a space-filling mode to evidence their location. Label 'eq' refers to the system conformation obtained after equilibration at $300 \mathrm{~K}$ and a very short simulation $(0.5 \mathrm{ps})$ at the chosen temperature. To make figures more readable, only molecules and ions in the residual nanodroplet are displayed. This figure is available in colour online at wileyonlinelibrary.com/journal/jms

ions are ejected individually, being surrounded by a shell of solvent molecules. All these out-of-equilibrium processes change the initial spherical contour of the droplet causing time-dependent protrusions and depressions of its surface and imparting an elongated shape.

With the necessary caution, due to significant deviation from spherical shape of the systems during the simulation, in Fig. 3, one may consider the radial number density of some relevant atomic species: oxygen atoms of water, sodium ions, sulfur atoms of the AOT head groups and one of the terminal carbon (C9) atoms of AOT tail. In all cases, the general picture suggested by these data is that the AOTNa molecules progressively selfrearrange as reverse micelle-like aggregates. A more detailed analysis of the panels of the figure shows that:

(1) Water oxygen atoms are initially located in the outer region of the nanodroplet according to a wide distribution centered at ca. $20 \AA$. After $1 \mathrm{~ns}$, at $\mathrm{T}=350 \mathrm{~K}$, they are mostly distributed at 1-10 $\AA$ from the center of the aggregate. The same behavior is evident at $500 \mathrm{~K}$, where the shrinkage of the droplet due to water expulsion is more evident. The water oxygen density vanishes at $800 \mathrm{~K}$ since at $1 \mathrm{~ns}$, practically all water molecules have left the initial droplet.

(2) The $\mathrm{Na}^{+}$ions are subject to a different but related fate: at 350 $\mathrm{K}$, they preferentially cluster in locations characterized by one or two distances from the aggregate center, after moving from the various positions they occupy initially within the nanodroplet. This is more evident at $500 \mathrm{~K}$, due to higher aggregation of the AOTNa molecules, and even more striking at $800 \mathrm{~K}$. For the latter case, where at $1 \mathrm{~ns}$ the fragmentation has already occurred, we consider the atom/ions distribution within the most populated AOTNa aggregate. (The number density reflects the fact that $\mathrm{Na}^{+}$ions left in the drop are found mostly in the wells formed by reverse micelle-type aggregates, the latter being quite small for $\mathrm{T}=800 \mathrm{~K}$ ).

(3) The $\mathrm{S}$ atoms behave similarly as the $\mathrm{Na}^{+}$ions: at $\mathrm{T}=350 \mathrm{~K}$, due to the presence of water, they are still removed from the center of the drop, at $\mathrm{T}=500 \mathrm{~K}$ and $\mathrm{T}=800 \mathrm{~K}$, instead, their distribution shows two well-defined distances near the center. This reflects the fact that at these temperature values, water molecules have mostly $(500 \mathrm{~K})$ or completely $(800 \mathrm{~K})$ diffused away favoring the formation of reverse micelle-like structures: the more anhydrous is the aggregate, the more solid-like is the core.

(4) The C9 atoms are initially located close to the droplet center since aliphatic tails are all entangled in a direct-micelle like aggregate. At $t=1 \mathrm{~ns}$ instead and for all $\mathrm{T}$ values, they fluctuate far from the initial positions and are found between 10 and $20 \AA$ from the center. For $\mathrm{T}=800 \mathrm{~K}$, these distances at $\mathrm{t}=1 \mathrm{~ns}$ are shorter since these data only refer to the largest AOTNa fragment.

Further information on the $[10,20]$ system can be gained from Fig. 4, where we illustrate the time dependence of the number of water molecules $\left(N_{W}\right)$, surfactant anions $\left(N_{A O T}\right)$ and sodium ions $\left(N_{N a}\right)$ belonging to the residual aggregate(s).

We observe that $N_{W}$ decreases linearly with time at $350 \mathrm{~K}$ indicating that a simulation time longer than 50 ns would be required to obtain anhydrous surfactant aggregates. Instead, at $500 \mathrm{~K}$ and $800 \mathrm{~K}, N_{W}$ decreases roughly exponentially giving rise to anhydrous aggregates within about 1 ns and 0.3 ns, respectively (see below). 

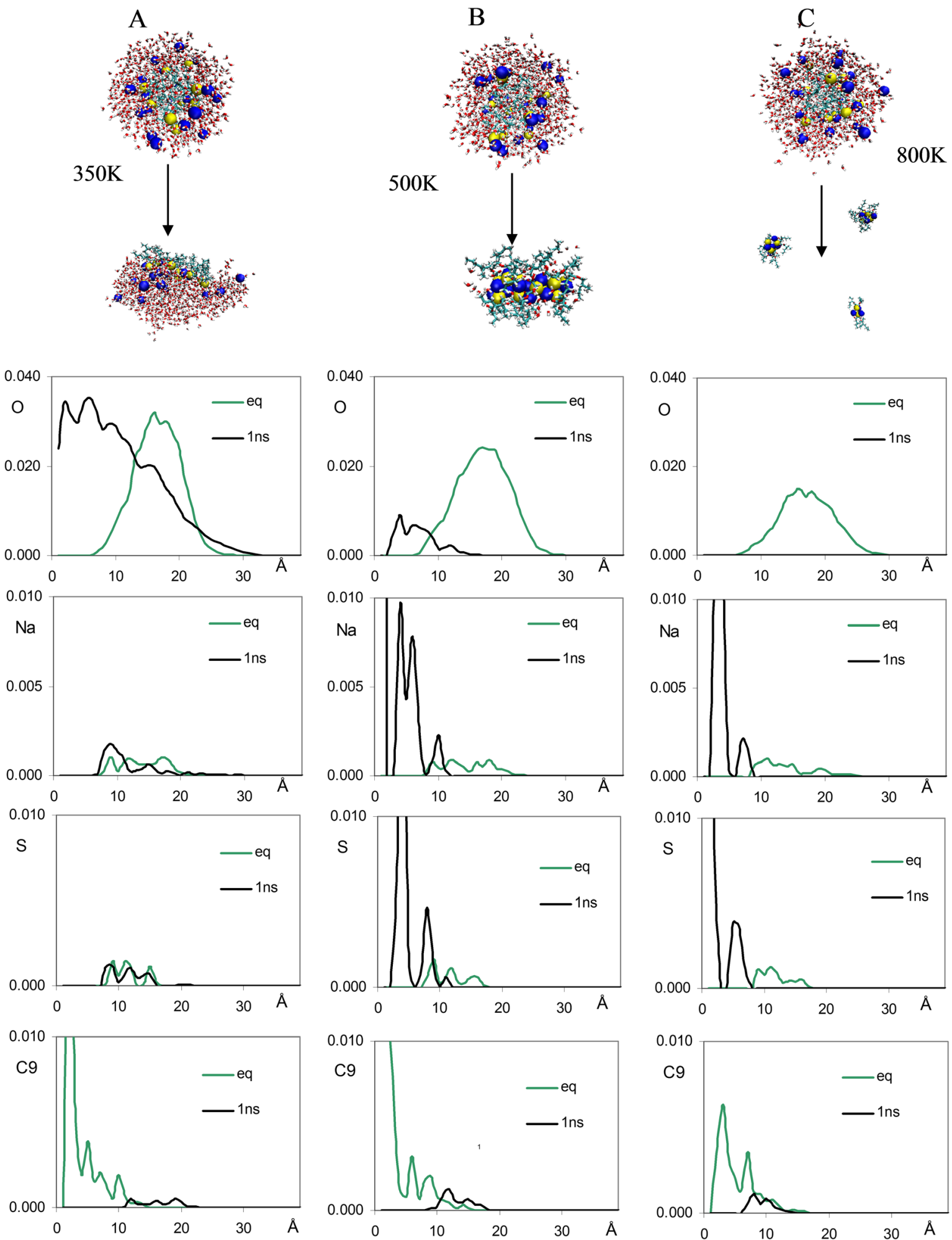

Figure 3. Snapshots of the $[10,20]$ systems at $350 \mathrm{~K}(\mathrm{~A}), 500 \mathrm{~K}(\mathrm{~B}), 800 \mathrm{~K}(\mathrm{C})$, and radial number density of water oxygen atoms (O), sodium ions (Na) and sulfur atoms (S) and terminal carbons (C9) of AOT: comparison between the situation at $1 \mathrm{~ns}$ and just after equilibration (see caption to Figure 2). In the snapshots, sodium ions (blue) and sulfur atoms of $\mathrm{SO}_{3}^{-}$polar heads (yellow) are displayed in a space-filling mode to evidence their location. This figure is available in colour online at wileyonlinelibrary.com/journal/jms

Ejection of sodium ions begins with some delay with respect to water evaporation, and there are time intervals when no sodium ion is ejected from the nanodroplet (see also Fig. 2). This behavior suggests that sodium ions are bound to the droplet, and their emission takes place when the charge density reaches some critical value. For this reason, we also evaluated the volume charge density (i.e. the ratio between the net charge and the volume of the residual droplet in $e / \AA^{3}$ units) versus time. We have computed the ratio of the sum of charges to the sum of volumes of all fragments generated by fragmentation and containing at least one AOTNa 


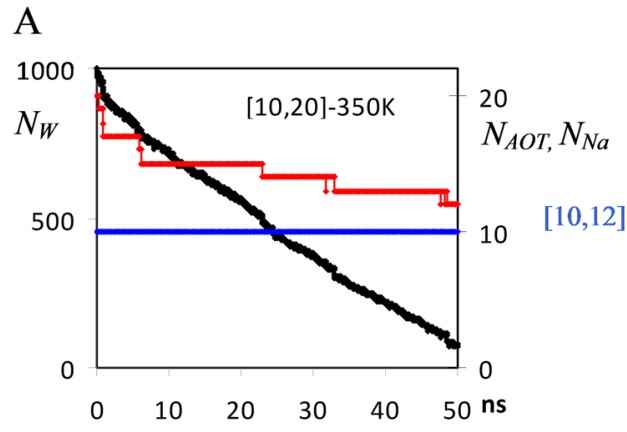

B
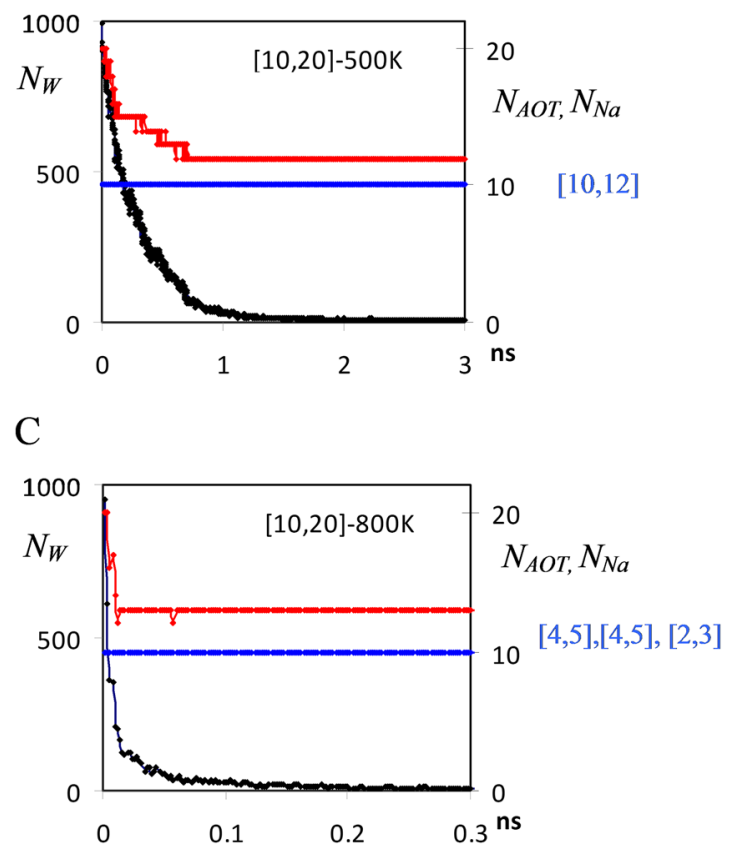

Figure 4. Time evolution of $N_{W}$ (left scale, black line), $N_{A O T}$ (right scale, blue line) and $N_{N a}$ (right scale, red line) of the $[10,20]$ system at $350 \mathrm{~K}(\mathrm{~A}), 500 \mathrm{~K}$ (B) and $800 \mathrm{~K}$ (C). On the right side of each panel, the final composition of the surfactant aggregate(s) is shown. This figure is available in colour online at wileyonlinelibrary.com/journal/jms

molecule. We report in Fig. 5 the time evolution of such charge density values for the $[10,20]$ system at the three temperatures considered. Since the total nanodroplet(s) volume decreases with time caused by water loss, charge density increases until a critical value of about $4-6 \cdot 10^{-4} e / \AA^{3}$ is reached, leading to ejection of a sodium ion. Indeed a slight, yet steady increase of the charge density to a plateau value is noted in panels B and C of Fig. 5. Some spikes in the charge densities are noticed in correspondence of the discontinuities of the number of $\mathrm{Na}$ atoms, at the instant they are ejected, causing at the same time a sudden change of aggregate charge state. During $\mathrm{Na}^{+}$ejection, the resulting decrease of the charge density of the residual nanodroplet implies that further water molecules are lost to induce further sodium expulsion. It is worth noting that a significantly higher charge density value is reached at $800 \mathrm{~K}$ due to aggregate fragmentation and involving a higher capacity to store net charges in separate AOTNa clusters.

It is also instructive to compare these trends with the ones predicted by the Rayleigh Eqn (1) assuming a nearly spherical shape for the droplets and deriving the needed $\mathrm{R}$ versus time from the volume analysis of MD trajectories. The charge density, reported in Fig. 6B as green lines for the $350 \mathrm{~K}$ case, is then
A

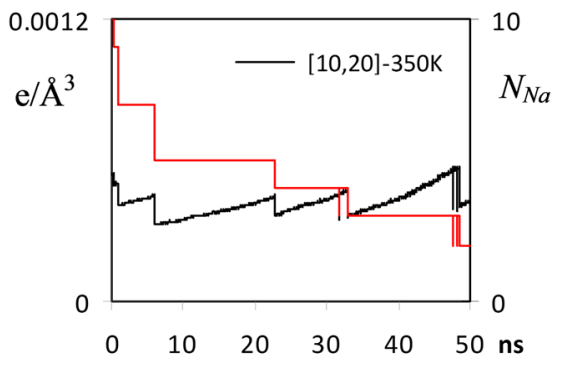

B

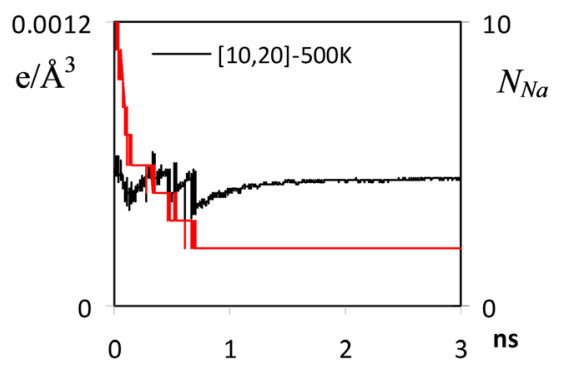

C

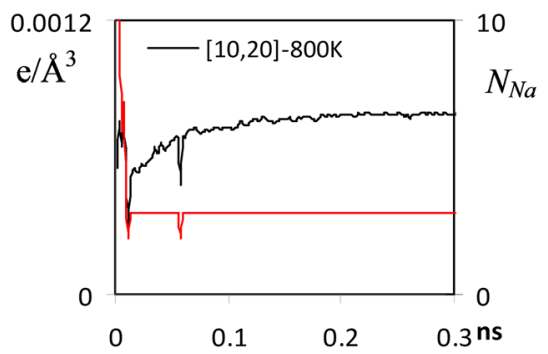

Figure 5. Time evolution of charge density (left scale, black line) and $N_{\mathrm{Na}}$ (right scale, red line) of the [10,20] system at $350 \mathrm{~K} \mathrm{(A),500} \mathrm{K} \mathrm{(B)} \mathrm{and} 800 \mathrm{~K}$ (C). This figure is available in colour online at wileyonlinelibrary.com/journal/jms

A

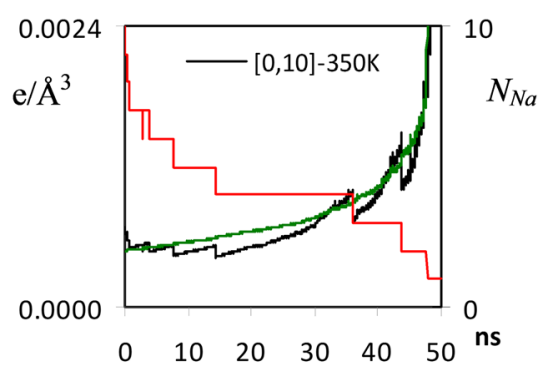

B

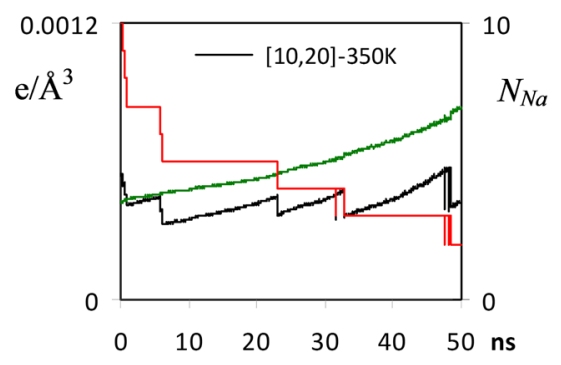

Figure 6. Time evolution of charge density (left scale, black line) and $N_{N a}$ (right scale, red line) of the $[0,10]$ system $(A)$ and the $[10,20]$ system $(B)$ at $350 \mathrm{~K}$. Green trace, charge density calculated from Rayleigh Eqn (1), as explained in the text. This figure is available in colour online at wileyonlinelibrary.com/journal/jms 
evaluated by assuming the $\gamma$ value of water at $350 \mathrm{~K}$. For this reason, the agreement is better for the pure water $[0,10]$ case, as illustrated by Fig. 6A. Taking into account that the Rayleigh equation is obtained considering macroscopic droplets with a continuous charge smeared on the droplet surface, the semi-quantitative agreement of simulation data with Rayleigh equation for nanoscopic water droplets is surprisingly good, suggesting its validity also for nano-sized droplets.

In order to evidence the fragmentation characteristics of the AOTNa aggregates occurring at $\mathrm{T}=800 \mathrm{~K}$, we report in Fig. 7 the numbers $N_{A O T}$ and $N_{N a}$ as functions of time for all individual clusters. Of course, the threshold used to establish fragmentation is crucial: in Fig. 2C, for example three clusters can be distinguished

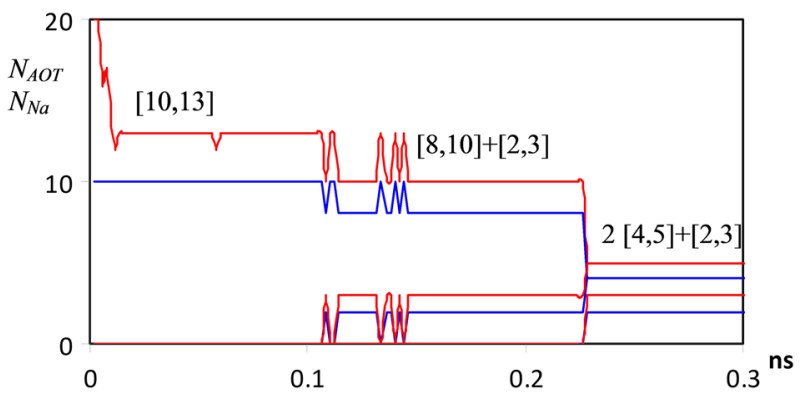

Figure 7. Time evolution of the fragments composition of the $[10,20]$ system at $800 \mathrm{~K}: N_{A O T}$ (blue line) and $N_{N a}$ (red line). This figure is available in colour online at wileyonlinelibrary.com/journal/jms at $\mathrm{t}=0.1 \mathrm{~ns}$, but two of them are located quite close to each other and are counted as a single larger $[8,10]$ cluster, consisting of 8 AOT $^{-}$ and $10 \mathrm{Na}^{+}$ions. The situation persists until ca. $0.2 \mathrm{~ns}$, and finally the two domains separate irreversibly.

Time dependence of $N_{W}, N_{A O T}$ and $N_{N a}$ and of charge density for the other examined systems are reported in the Supplementary Information file (SI). Relevant data are also collected in Table 1, in order to make comparison of their characteristics easier and to suggest possible new experiments to be performed. In particular, we report the composition of the nanodroplets in terms of $\left[N_{A O T}, N_{N a}\right]$ aggregate(s) and of residual water molecules at the end of the simulation (i.e. $50 \mathrm{~ns}$ for systems at $350 \mathrm{~K}$ and $3 \mathrm{~ns}$ for systems at $500 \mathrm{~K}$ and $800 \mathrm{~K}$ ), the value $t_{1 / 2}$ of the time at which half of the initial water molecules has left the droplet, the fraction $\mathrm{f}_{\mathrm{w}}{ }^{*}$ of the water molecules still present in the droplet at the time when the first $\mathrm{Na}^{+}$ion is ejected and the charge density $\rho^{*}$ of the droplet at the same time.

From Table 1, one can see that for all systems at $350 \mathrm{~K}$, one aggregate is obtained at the end of each 50-ns simulation, which includes the same number of surfactant molecules present in the initial droplet. This is also true for the 3-ns simulations at $500 \mathrm{~K}$, whereas for those at $800 \mathrm{~K}$, the droplets end up very frequently with the fragmentation in smaller surfactant aggregates, in agreement with previous findings. ${ }^{[24]}$

As expected, $t_{1 / 2}$ depends on temperature to a major extent, but depends only slightly on the charge state of the droplet, suggesting that the ion-ion interactions are shielded by water. The rather strong interaction between $\mathrm{Na}^{+}$ions and water is also

Table 1. Relevant parameters constructed from the simulations of the $\left[N_{A O T}^{0}, N_{N a}^{0}\right]$ systems: aggregate composition $\left[N_{A O T}, N_{N a}\right]$ at the end of the simulation (i.e. $50 \mathrm{~ns}$ at $350 \mathrm{~K} ; 3 \mathrm{~ns}$ at 500 and $800 \mathrm{~K}$ ), with residual number of water molecules indicated in round brackets; time $t_{1 / 2}$ (ns) when $N_{W}=$ 500 (water molecules' half-life); fraction of water molecules $\mathrm{fw}_{\mathrm{w}}{ }^{*}$ and corresponding charge density $\rho^{*} \times 10^{3}\left(\mathrm{e} / \AA^{3}\right.$ ) of the nanodroplet when the first $\mathrm{Na}^{+}$is ejected

\begin{tabular}{|c|c|c|c|c|c|}
\hline System & $\mathrm{T}(\mathrm{K})$ & Aggregate/(s) composition & $t_{1 / 2}$ & $f_{W}^{*}$ & $\rho^{*}$ \\
\hline \multirow[t]{3}{*}[10,20]{} & 350 & {$[10,12](76)$} & 22.9 & 0.98 & 0.54 \\
\hline & 500 & {$[10,12](4)$} & 0.17 & 0.80 & 0.63 \\
\hline & 800 & {$[4,5],[4,5],[2,3]$} & 0.0032 & 0.61 & 0.76 \\
\hline \multirow[t]{3}{*}[10,15]{} & 350 & {$[10,13](110)$} & 26.0 & 0.54 & 0.43 \\
\hline & 500 & {$[10,12](5)$} & 0.21 & 0.48 & 0.46 \\
\hline & 800 & {$[5,6],[2,3](1),[2,3],[1,2](2)$} & 0.0029 & 0.17 & 0.80 \\
\hline \multirow[t]{3}{*}[10,10]{} & 350 & {$[10,10](144)$} & 30.8 & - & - \\
\hline & 500 & {$[10,10]$} & 0.26 & - & - \\
\hline & 800 & {$[8,8],[2,2]$} & 0.0031 & - & - \\
\hline \multirow[t]{3}{*}[5,15]{} & 350 & {$[5,7](53)$} & 23.2 & 0.99 & 0.60 \\
\hline & 500 & {$[4,5] 2 w,[1,2](7)$} & 0.16 & 0.89 & 0.65 \\
\hline & 800 & {$[3,4],[2,3]$} & 0.0029 & 0.79 & 0.72 \\
\hline \multirow[t]{3}{*}[5,10]{} & 350 & {$[5,7](83)$} & 25.0 & 0.49 & 0.54 \\
\hline & 500 & {$[5,6](2)$} & 0.21 & 0.58 & 0.47 \\
\hline & 800 & {$[4,5],[1,2]$} & 0.0027 & 0.46 & 0.56 \\
\hline \multirow[t]{3}{*}[5,5]{} & 350 & {$[5,5](157)$} & 26.3 & - & - \\
\hline & 500 & {$[5,5](1)$} & 0.24 & - & - \\
\hline & 800 & {$[5,5]$} & 0.0036 & - & - \\
\hline \multirow[t]{3}{*}[0,10]{} & 350 & {$[0,1](13)$} & 22.2 & 0.99 & 0.67 \\
\hline & 500 & {$[0,1](6)$} & 0.18 & 0.80 & 0.82 \\
\hline & 800 & {$[0,1](2)$} & 0.0031 & 0.65 & - \\
\hline \multirow[t]{3}{*}[0,5]{} & 350 & {$[0,2](75)$} & 25.2 & 0.52 & 0.64 \\
\hline & 500 & {$[0,1](5)$} & 0.22 & 0.46 & 0.70 \\
\hline & 800 & {$[0,1](2)$} & 0.0031 & 0.32 & - \\
\hline \multirow[t]{2}{*}[0,0]{} & 350 & {$[0,0](141)$} & 26.4 & - & - \\
\hline & 500 & {$[0,0](1)$} & 0.25 & - & - \\
\hline
\end{tabular}


suggested by Fig. 2, where one may notice that $\mathrm{Na}^{+}$ions leave the droplet being surrounded by a number of water molecules.

Sodium ion emission is observed when the droplet charge density is about 4-6 $6 \cdot 10^{-4} \mathrm{e} / \AA^{3}$ which approximately corresponds to an electric $E$ field intensity at the surface of $2-5 \mathrm{~V} / \mathrm{nm}$, as determined by assuming a spherical shape of the nanodroplet. This value is consistent with the minimum field strength at the droplet surface producing ion emission (of the order of $1 \mathrm{~V} / \mathrm{nm}$ ), as evaluated by Iribarne and Thomson. ${ }^{[7,39,40]}$ These Authors described the process as determined by the combination of the solvation energy of the evaporated ion and by electrostatic interaction between the ion and the droplet.

No sodium ion emission is observed in the case of neutral droplets, whereas the rate of ejection of $\mathrm{Na}^{+}$ions significantly increases with the droplet net charge, as indicated by the $f_{W}{ }^{*}$ value and as documented by the plots reported in Supporting Information. Interestingly, ion emission increases with temperature before fragmentation and decreases after fragmentation has taken place. It is worth noting that aggregates maintain a higher charge state at low temperature and when they are formed from droplets with high initial charge (i.e. higher potential attached to the capillary and/or higher concentration of ions in the starting solution).

As shown in Table 1, the value of water fraction $f_{W}{ }^{*}$ still present in the droplet when the first $\mathrm{Na}^{+}$ion is ejected decreases by increasing temperature, implying that a temperature increase enhances the emission rate for water molecules more than for $\mathrm{Na}^{+}$ions. This again speaks in favour of a strong ion-water interaction. Moreover, at fixed charge state, a higher number of AOTNa molecules in the droplet involves a stabilization of the extra charges, making the ion emission more difficult.

\section{Conclusions}

The behavior of aqueous nanodroplets containing few AOT $^{-}$and $\mathrm{Na}^{+}$ions has been investigated by MD simulation, with the aim to mimic AOTNa solutions under ESI conditions. Taking into account that the typical residence time of charged species within the mass spectrometer is of the order of $10 \mathrm{~ms}^{[1]}$ our results indicate that in the ESI positive mode the surfactant aggregates produced from aqueous nanodroplets, after complete water evaporation and loss of some sodium ions, are expected to be experimentally observed. The size distribution of detectable aggregates results from the statistical averages of surfactant molecules over the various nanodroplets initially produced by the ESI apparatus. Furthermore, the simulations show that only at sufficiently high temperature fragmentations occur and aggregates smaller than those observed at low temperature should be detected by ESI-MS experiments.

All performed simulations also show that surfactant AOTNa molecules, initially organized as direct micelles in the aqueous solution, self-reassemble in ESI conditions as reverse micellelike aggregates in a very short time. This finding is of utmost importance in view of the possible use of surfactant aggregates in the gas phase for transporting and protecting drugs or other biomolecules to the lungs, and/or as nanoreactors for specialized reaction in confined space and atmospheric cleaning agents. Moreover, the present results may shed some light onto the studies of molecular aggregates in the interstellar space and may be relevant for researches about the origin of life.

Finally, our simulations show that sodium ions are emitted from aqueous nanodroplets as solvated species when the charge density reaches a critical value. This implies that, consistent with experimental data, ${ }^{[41]}$ a higher charge state should be observed for larger aggregates. Moreover, since the electric field at the droplet surface needed to cause sodium ion ejection is four orders of magnitude larger than the typical externally applied electric field (about $5 \mathrm{kV} / \mathrm{cm}$ ), we may argue that the latter field has no effect on the droplet time evolution.

\section{Acknowledgement}

Financial support from the Italian Ministero dell'Istruzione dell'Università e della Ricerca (MIUR) is gratefully acknowledged.

\section{Supporting information}

Supporting information may be found in the online version of this article.

\section{References}

[1] M. Yamashita, J. B. Fenn. Electrospray Ion Source. Another Variation on the Free-Jet Theme. J. Phys. Chem. 1984, 88, 4451.

[2] S. Crotti, R Seraglia, P Traldi, Some thoughts on electrospray ionization mechanisms. Eur. J. Mass Spectrom. 2011, 17, 85.

[3] P. Pan, S. A. McLuckey, Electrospray lonization of Protein Mixtures at Low pH. Anal. Chem. 2003, 75, 1491.

[4] D. Bongiorno, L. Ceraulo, G. Giorgi, S. Indelicato, M. Ferrugia, A. Ruggirello, V. Turco Liveri. Effects of the net charge on abundance and stability of supramolecular surfactant aggregates in gas phase J. Mass. Spectrom. 2011, 46, 195.

[5] J. F. De La Mora. On the outcome of the Coulomb fission of a charged isolated drop. J. Colloid Interface Sci., 1996, 178, 209.

[6] P. Kebarle. A brief overview of the present status of the mechanisms involved in electrospray mass spectrometry. J. Mass Spectrom. 2000, 35, 804.

[7] W. D. Luedtke, U. Landman, Y.-H. Chiu, D. J. Levandier, R. A. Dressler, S. Sok, M. S. Gordon. Nanojets, electrospray, and ion field evaporation: molecular dynamics simulations and laboratory experiments J. Phys. Chem. A 2008, 112, 9628.

[8] V. Znamenskiy, I. Marginean, A. Vertes, Solvated Ion Evaporation from Charged Water Nanodroplets. J. Phys. Chem. A 2003, 107, 7406.

[9] E. Ahadi, L. Konermann. Ejection of Solvated lons from Electrosprayed Methanol/Water Nanodroplets Studied by Molecular Dynamics Simulations. J. Am. Chem. Soc. 2011, 133, 9354.

[10] J. N. Smith, R. C. Flagan, J. L. Beauchamp. Droplet Evaporation and Discharge Dynamics in Electrospray lonization. J. Phys. Chem. A 2002, 106, 9957.

[11] E. Ahadi, L. Konermann. Molecular Dynamics Simulations of Electrosprayed Water Nanodroplets: Internal Potential Gradients, Location of Excess Charge Centers, and 'Hopping' Protons. J. Phys. Chem. B 2009, 113, 7071.

[12] I. Marginean, V. Znamenskiy, A. Vertes. Charge reduction in electrosprays: slender nanojets as intermediates. J. Phys. Chem. B 2006, 110, 6397.

[13] K. Ichiki, S. Consta. Disintegration mechanisms of charged aqueous nanodroplets studied by simulations and analytical models. J. Phys. Chem. B 2006, 110, 19168.

[14] J. T. Zhang, B. X. Wang. Study on the interfacial evaporation of aqueous solution of SDS surfactant self-assembly monolayer. Int. J. Heat and Mass Trans. 2003, 46, 5059.

[15] J. W. S. Lord Rayleigh. On the Equilibrium of Liquid Conducting Masses Charged with Electricity. Phil. Mag. 1882, 14, 184.

[16] D. Myers. Surfaces, Interfaces, and Colloids. Principles and applications Ed Wiley-VCH, NY, 1999 ISBN 0-471-33060-4.

[17] D. van der Spoel, E. G. Marklund, D. S. D. Larsson, C. Caleman. Proteins, Lipids, and Water in the Gas Phase. Macromol. Biosci. 2011, 11, 50.

[18] Y. Wang, D. S. D. Larsson, D. van der Spoel. Encapsulation of myoglobin in a cetyl trimethylammonium bromide micelle in vacuo: a simulation study. Biochemistry 2009, 48, 1006.

[19] D. Bongiorno, L. Ceraulo, A. Ruggirello, V. Turco Liveri, E. Basso, R. Seraglia, P. Traldi. Surfactant self-assembling in gas phase: 
electrospray ionization and matrix-assisted laser desorption/ionization-mass spectrometry of singly charged AOT clusters. J. Mass Spectrom. 2005, 40, 1618.

[20] D. Bongiorno, L. Ceraulo, G. Giorgi, S. Indelicato, A. Ruggirello, V. Turco Liveri. Supramolecular aggregates in vacuum: positively monocharged sodium alkanesulfonate clusters. Eur. J. Mass Spectrom. 2010, 16, 151.

[21] Y. Fang, A. Bennett, J. Liu. Multiply charged gas-phase NaAOT reverse micelles: Formation, encapsulation of glycine, and collisioninduced dissociation. Int. J. Mass Spectrom. 2010, 293, 12.

[22] Y. Fang, A. Bennett, J. Liu. Selective transport of amino acids into the gas phase: Driving forces for amino acid solubilization in gas-phase reverse micelles. Phys. Chem. Chem. Phys., 2011, 13, 1466.

[23] G. Longhi, S. L. Fornili, V.Turco Liveri, S. Abbate, D. Rebeccani, L. Ceraulo, F. Gangemi, Sodium bis(2-ethylhexyl)sulfosuccinate selfaggregation in vacuo: molecular dynamics simulation. Phys. Chem. Chem. Phys. 2010, 12, 4694.

[24] G. Longhi, S. Abbate, L. Ceraulo, A. Ceselli, S. L. Fornili, V. Turco Liveri. A molecular dynamics study of structure, stability and fragmentation patterns of sodium bis(2-ethylhexyl)sulfosuccinate positively charged aggregates in vacuo. Phys. Chem. Chem. Phys. 2011, 13, 21423.

[25] F. Heatley. A $1 \mathrm{H}$ and $13 \mathrm{C}$ nuclear magnetic resonance study of the conformation of aerosol OT in water and hydrocarbon solutions. J Chem Soc Faraday Trans I 1987, 83, 517.

[26] D. Bongiorno, L. Ceraulo, G. Giorgi, S. Indelicato, V. Turco Liveri. Do electrospray mass spectra of surfactants mirror their aggregation state in solution? J. Mass Spectrom. 2011, 46, 1262.

[27] L. Bijlsma, J. V. Sancho, E. Pitarch, M. Ibánez, F. Hernández. Simultaneous ultra-high-pressure liquid chromatography-tandem mass spectrometry determination of amphetamine and amphetamine-like stimulants, cocaine and its metabolites, and a cannabis metabolite in surface water and urban wastewater. J. Chromatogr. A 2009, 1216, 3078

[28] J. Chico, A. Rúbies, F. Centrich, R. Companyó, M. D. Prat, M. Granados, High-throughput multiclass method for antibiotic residue analysis by liquid chromatography-tandem mass spectrometry. J. Chromatogr. A 2008, 1213, 189 .
[29] J. Wang, R. M. Wolf, J. W. Caldwell, P. A. Kollman, D. A. Case. Development and testing of a general amber force field. J. Comput. Chem., 2004, 25, 1157.

[30] C. I. Bayly, P. Cieplak, W. Cornell, P. A. Kollman. A well-behaved electrostatic potential based method using charge restrains for deriving atomic charges. The RESP model. J. Phys. Chem., 1993, 97, 10269.

[31] D. A. Case, T. A. Darden, T. E. Cheatham, III, C. L. Simmerling, J. Wang, R. E. Duke, R. Luo, M. Crowley, R. C. Walker,W. Zhang, K. M. Merz, B. Wang, S. Hayik, A. Roitberg, G. Seabra, I. Kolossváry, K. F. Wong, F. Paesani, J. Vanicek, X. Wu, S. R. Brozell, T. Steinbrecher, H. Gohlke, L. Yang, C. Tan, J. Mongan, V. Hornak, G. Cui, D. H. Mathews, M. G. Seetin, C. Sagui, V. Babin, P. A. Kollman. AMBER 10, University of California, San Francisco, (2008).

[32] W. L. Jorgensen, J. Chandrasekhar, J. D. Madura, R. W. Impey, M. L. Klein. Comparison of simple potential functions for simulating liquid water. J. Chem. Phys. 1983, 79, 926.

[33] D. A. Case, T. E. Cheatham, III, T. Darden, H. Gohlke, R. Luo, K. M. Merz, Jr., A. Onufriev, C. Simmerling, B. Wang, R. Woods. The AMBER biomolecular simulation programs. J. Comput. Chem., 2005, 26, 1668

[34] M. P. Allen, T. J. Tildesley. Computer Simulation of Liquid. Clarendon Press: Oxford, 1987.

[35] J. C. Phillips, R. Braun, W. Wang, J. Gumbart, E. Tajkhorshid, E. Villa, C. Chipot, R. D. Skeel, L. Kalé, K. Schulten. Scalable molecular dynamics with NAMD. J. Comput. Chem. 2005, 26, 1781.

[36] W. Humphrey, A. Dalke, K. Schulten. VMD: Visual molecular dynamics. J. Mol. Graphics 1996, 14, 33.

[37] A. Balaeff [http://www.ks.uiuc.edu/Development/MDTools/molvolume/].

[38] D. E. Knuth. The art of computer programming, vol.1. Addison-Wesley: Boston, 1997.

[39] J. V. Iribarne, B. A. Thomson. On the evaporation of small ions from charged droplets. J. Chem. Phys. 1976, 64, 2287.

[40] M. Gamero-Castano1, J. Fernandez de la Mora. Kinetics of small ion evaporation from the charge and size distributions of multiply charged electrospray clusters. J. Mass Spectrom.. 2000, 35, 790.

[41] A. J. Borysik, C. V. Robinson. Formation and dissociation processes of gas-phase detergent micelles. Langmuir 2012, 28, 7160. 